\title{
Ultra-Mutation in IDH Wild-Type Glioblastomas of Patients Younger than 55 Years is Associated with Defective Mismatch Repair, Microsatellite Instability, and Giant Cell Enrichment
}

\author{
Valeria Barresi ${ }^{1, *,+} \oplus$, Michele Simbolo ${ }^{1,+} \oplus$, Andrea Mafficini ${ }^{2}$, Maria Liliana Piredda ${ }^{1}$, \\ Maria Caffo ${ }^{3}$, Salvatore Massimiliano Cardali ${ }^{3}$, Antonino Germanò ${ }^{3}$, Sara Cingarlini ${ }^{4}$, \\ Claudio Ghimenton ${ }^{5}$ and Aldo Scarpa ${ }^{1,2,5,+}$ \\ 1 Department of Diagnostics and Public Health, Section of Anatomical Pathology, University and Hospital \\ Trust of Verona, 37134 Verona, Italy \\ 2 ARC-Net Research Centre, University and Hospital Trust of Verona, 37134 Verona, Italy \\ 3 Department of Biomedical and Dental Sciences and Morphofunctional Imaging, Section of Neurosurgery, \\ University of Messina, 98125 Messina, Italy \\ 4 Department of Medicine, Section of Medical Oncology, University and Hospital Trust Verona, 37134 Verona, \\ Italy \\ 5 Department of Pathology and Diagnostics, Section of Pathology, Hospital Trust Verona, 37134 Verona, Italy \\ * Correspondence: valeria.barresi@univr.it; Tel.: +39-045-8127728 \\ + These authors contributed equally to this paper.
}

Received: 8 August 2019; Accepted: 28 August 2019; Published: 30 August 2019

check for updates

\begin{abstract}
Background: Glioblastomas (GBMs) are classified into isocitrate dehydrogenase (IDH) mutants and $I D H$ wild-types (IDH-wt). This study aimed at identifying the mutational assets of $I D H$-wt GBMs in patients aged 18-54 years for which limited data are available. Methods: Sixteen $I D H$-wt GBMs from adults $<55$ years old were explored for mutations, copy number variations, tumour mutational load (TML), and mutational spectrum by a 409 genes TML panel. Results: Eight $(50 \%)$ IDH-wt GBMs were hypermutated (TML $>9$ mutations/Mb) and two (12.5\%) were ultra-mutated (TML > 100 mutations/Mb). One ultra-mutated GBM had microsatellite instability (MSI), a somatic MSH6 mutation, and a germline POLE mutation. The other ultra-mutated GBMs had MSI and two somatic mutations in MSH2. Both ultra-mutated GBMs featured at least $25 \%$ giant cells. The overall survival of eight patients with hypermutated GBMs was significantly longer than that of patients with non-hypermutated GBMs $(p=0.04)$. Conclusions: We identified a hyper-mutated subgroup among $I D H$-wt GBMs in adults $<55$ years that had improved prognosis. Two cases were ultra-mutated and characterized by the presence of at least $25 \%$ giant cells, MMR mutations, and MSI. Since high TML has been associated with response to immune checkpoint inhibition in paediatric gliomas, the identification of a subtype of ultra-mutated IDH-wt GBM may have implications for immunotherapy.
\end{abstract}

Keywords: glioblastoma; IDH wild-type; giant cells; mismatch repair; POLE; tumour mutation load

\section{Introduction}

Glioblastoma (GBM) is the most common malignant primary tumour of the central nervous system in adults [1]. The standard of care for patients affected by GBM is maximal safe surgical resection, followed by chemotherapy with temozolomide and radiotherapy [2,3]. However, despite treatment, most GBM patients undergo recurrence and die within 15-18 months of diagnosis, while only about $5 \%$ survive more than 5 years [1]. 
The 2016 World Health Organization (WHO) classification subdivides GBM into isocitrate dehydrogenase $(I D H)$ mutant and IDH wild-type (wt), based on the mutational status of IDH1/IDH2 genes [4]. This distinction is prognostically relevant. Patients with IDH-mutant GBM have significantly longer overall and recurrence-free survival compared with patients with IDH-wt GBM [4-6]. Moreover, these two types of GBM have different age distributions and pathogenesis.

The IDH-mutant GBM is more frequent in younger patients ( $<55$ years, median: 45 years) and is considered to be secondary, i.e., derived from the progression of low-grade astrocytoma. In addition, it is characterized by frequent mutations in the ATRX and TP53 genes [4].

The IDH-wt GBM mainly affects older subjects (> 55 years, median: 62 years) [7] and is considered primary, i.e., arises de novo. From a molecular standpoint, $I D H$-wt GBM is defined by the absence of $I D H$ mutations and may have different genetic alterations. Among those, EGFR amplification is the most frequent (35-45\%), followed by PTEN mutations/deletions and CDKN2A deletions [8-11].

In a recent study, about $25 \%$ of GBMs, and mainly $I D H$-wt ones, displayed microsatellite instability (MSI) [12]. This is a condition of genetic hypermutability resulting from defective DNA mismatch repair (MMR) and characterized by clustering of mutations in highly repetitive short DNA sequences, called microsatellites [13]. Microsatellite instability is a marker of defective MMR [12], which is mostly found in recurrent GBMs as a result of treatment [14].

As IDH-wt GBM has the lowest incidence between 18 and 55 years, most studies on IDH-wt GBM focused on either paediatric age or patients above 55 years $[5,9,15-19]$. Thus, the mutational spectrum of $I D H$-wt GBM has not been specifically investigated in adults between 18 and 54 years of age.

This study aimed to identify the mutational spectrum of $I D H$-wt GBMs from adult patients younger than 55 years, exploring mutations and copy number variations of 409 genes as well as tumour mutational load and mutational signatures.

\section{Results}

\subsection{Clinico-pathological Features}

Our cohort of IDH-wt GBMs included 16 cases out of 108 (14.8\%) consecutive GBMs that had been diagnosed in patients $<55$ years in a single hospital. The cohort comprised 5 female and 11 male patients (mean age 38.3 years; range: $18-49$ years) (Table 1 ).

One patient (case $5 \mathrm{GL}$ ) had a breast ductal carcinoma seven years before the occurrence of GBM. Brain metastatic carcinoma was excluded by immunohistochemistry with anti-GFAP and anti-cytokeratins antibodies. No other patient had or developed other tumours during follow-up. One patient (case $4 \mathrm{GL}$ ) had a family history (one brother) positive for GBM. The overall survival of the patients ranged between 1 and 79 months (median: 23.5 months; mean: 28.7 months).

At histopathology, one tumour (15 GL) was an epithelioid GBM (as defined by the presence of a dominant population of closely packed epithelioid cells) [10], one (5 GL) was a giant cell GBM (as defined by the presence of dominant bizarre multinucleated giant cells) [10], and three additional cases (1 GL, 3 GL, 12 GL) contained at least 25\% multinucleated giant cells (Figure 1). In detail, in cases $1 \mathrm{GL}, 3 \mathrm{GL}$, and $12 \mathrm{GL}$, giant cells (respectively accounting for $50 \%, 50 \%$, and $25 \%$ of the tumour cells) were homogeneously distributed and intermingled with mononuclear tumour cells with milder atypia. Cases $9 \mathrm{GL}$ and $16 \mathrm{GL}$ also had giant cells representing less than $10 \%$ of cancer cells. 
Table 1. Clinico-pathological features and main molecular data of $16 \mathrm{IDH}$-wt GBMS in adult patients younger than 55 years. Cases are ordered by increasing TML.

\begin{tabular}{|c|c|c|c|c|c|c|c|c|c|c|c|}
\hline Case & Gender & Age & Site & $\begin{array}{c}\text { OS } \\
\text { (months) }\end{array}$ & IDH1/2 & EGFR & ATRX & TP53 & PTEN & TML & MMR Genes \\
\hline $4 \mathrm{GL}$ & M & 49 & fronto-temporal right & DOD (11) & wt & ampl & & & & 5.28 & \\
\hline $6 \mathrm{GL}$ & $\mathrm{M}$ & 41 & fronto-temporal right & $\mathrm{DOD}(3)$ & wt & ampl & & & & 5.66 & \\
\hline $2 \mathrm{GL}$ & $\mathrm{F}$ & 49 & temporo-parietal left & $\mathrm{DOD}(46)$ & wt & $\mathrm{ampl} / \mathrm{mut}$ & & & & 6.34 & \\
\hline $15 \mathrm{GL}$ & $\mathrm{M}$ & 43 & fronto-basal & DOD (14) & wt & & & mut & & 6.47 & \\
\hline $16 \mathrm{GL}$ & M & 40 & temporal left & $\operatorname{DOD}(9)$ & wt & & & & mut & 7.12 & \\
\hline $7 \mathrm{GL}$ & M & 39 & temporo-parieto-occipital left & DOD (14) & wt & ampl & & & & 7.33 & \\
\hline $13 \mathrm{GL}$ & $\mathrm{F}$ & 41 & temporal left & Alive (24) & wt & ampl & & & & 7.75 & \\
\hline $9 \mathrm{GL}$ & $\mathrm{M}$ & 18 & temporo-parietal left & Alive (38) & wt & & & mut & mut & 8.22 & \\
\hline $11 \mathrm{GL}$ & $\mathrm{F}$ & 29 & cerebellar right & DOD (15) & wt & & & & & 9.24 & \\
\hline $8 \mathrm{GL}$ & M & 46 & temporo-parietal left & Alive (41) & $\mathrm{wt}$ & & & & mut & 12.35 & \\
\hline $1 \mathrm{GL}$ & $\mathrm{F}$ & 47 & temporo-parieto-occipital right & Alive (23) & wt & & & mut & mut & 13.07 & MLH1 mut ${ }^{*}$ \\
\hline $14 \mathrm{GL}$ & $\mathrm{M}$ & 43 & frontal lobe & Alive (14) & wt & & & mut & & 14.34 & \\
\hline $3 \mathrm{GL}$ & M & 24 & temporal left & Alive (24) & wt & $\mathrm{ampl} / \mathrm{mut}$ & & mut & & 43.19 & \\
\hline $12 \mathrm{GL}$ & $\mathrm{M}$ & 39 & frontal & Alive (32) & wt & & mut & mut & & 168.02 & MSH6 mut \\
\hline $5 \mathrm{GL}$ & $\mathrm{F}$ & 37 & frontal & Alive (79) & wt & & & mut & mut & 219.79 & MSH2 mut \\
\hline
\end{tabular}

OS: overall survival. DOD: died of disease. TML: tumour mutation load. MMR: mismatch repair. F: female. M: male. Ampl: amplified. Mut: mutation. ${ }^{*}$ Classified as benign in the PolyPhen Database. 


\subsection{Mutational Status of 409 Genes}

All 16 cases were analysed for 409 genes included in the TML assay panel (ThermoFisher, Waltham, MA, USA). Sequencing achieved an average coverage of $282 \times(36 \times-712 \times)$ in tumours and $236 \times(26 \times-651 \times)$ in normal samples (Supplementary Materials Table S1). All cases were confirmed to be IDH1/2 wild-type. Mutations were found in at least one gene in 13 of the 16 cases, while three samples (7 GL, 11 GL, 13 GL) had no mutation in any of the 409 genes analysed (Figure 2, Supplementary Materials Table S2). Those latter cases had only mutations in untranslated regions. A total of 45 mutations in 29 genes were identified, including 19 missense, 16 nonsense, 7 frameshift, and 3 splice site alterations (Supplementary Materials Table S2).

The most frequent inactivating somatic mutations involved TP53 (7/16; 43.8\%) and PTEN (6/16; $37.5 \%$ ) genes, followed by MMR genes that were found altered in 3 GBMs: case 1 GL had a missense Ser193Leu mutation in MLH1 that is classified as benign in PolyPhen database, case 5 GL had two MSH2 mutations including a truncating Val684Ter and a missense Gly164Glu mutation, case 12 GL had a truncating Glu1322Ter mutation in MSH6. The EGFR activating Arg108Lys mutation was found in two cases (2 GL and 3 GL). A truncating Glu1365Ter mutation in the ATRX gene was found in one case (12 GL). A BRAF Val600Glu mutation was observed in the epithelioid GBM 15 GL. An FGFR1 pathogenic missense Lys687Asp mutation was seen in case 14 GL.

Germline heterozygous mutations were found in three patients, comprising the MUTYH Gly396Asp (rs36053993) in patients 6 GL and 12 GL and a stop-gain (Glu265Ter) in RNASEL (rs74315364) in patient 2 GL (Figure 1).

\subsection{Gene and Chromosomal Copy Number Alterations}

The CNV status was estimated for all 409 genes using sequencing data. Four genes had focal amplification: EGFR in 6/16 cases (37.5\%), CCNE1, CDK4, and MDM2 in 1 case each (6.3\%). Two genes showed homozygous deletion: $C D K N 2 A$ in $5 / 16$ cases (31.3\%) and $R B 1$ in 3/16 cases $R B 1$ in $3 / 16$ cases. Based on the chromosomal position of each gene, the status of chromosome arms was inferred (Figure 3). The major alterations were gains in chromosome 7 (14/16; 87.5\%) and losses in chromosomes $10(6 / 16 ; 37.5 \%), 13(12 / 16 ; 75.0 \%)$, and in $19 q$ arm $(11 / 16 ; 68.8 \%)$. Frequent homozygous deletions were detected in chromosome 19 and involved loci of BCL3, CIC, and MARK4 genes (6/16; 37.5\%).

\subsection{Tumour Mutational Load}

The number of mutations/Mb in the $16 \mathrm{GBMs}$ ranged from 5.28 to 219.79 (median 8.73) (Figure 2, Table 1); 8 of the 16 cases (1 GL, 3 GL, 5 GL, 8 GL, 10 GL, 11 GL, 12 GL, 14 GL) had > 9 muts/Mb and were considered hypermutated; 2 of these ( 5 GL and 12 GL) had $>100$ muts/Mb and were considered ultra-mutated according to Campbell et al. [20].

\subsection{Mutational Spectrum}

The mutational spectrum of our IDH-wt cohort was characterized by prevalent $\mathrm{T}>\mathrm{C}$ and $\mathrm{C}>\mathrm{T}$ transitions with low to absent contribution of $\mathrm{T}>\mathrm{A}, \mathrm{T}>\mathrm{G}, \mathrm{C}>\mathrm{G}$, and $\mathrm{C}>\mathrm{A}$ transversions (Figures 1 and 4). Notably, in addition to the predominance of $\mathrm{C}>\mathrm{T}$ over $\mathrm{T}>\mathrm{C}$ transitions, the ultra-mutated case 12 GL also showed a significant proportion of $\mathrm{C}>\mathrm{A}$ transversions (Figure 1), which presented four peaks in correspondence of the trinucleotide contexts CCT, TCT, GCT, and ACT (Figure 2). This C $>$ A mutational pattern accompanied to prevalent $\mathrm{C}>\mathrm{T}$ transitions corresponds to signature SBS14 in the COSMIC database [21], which has been associated with the concurrent impairment of POLE and MMR functions [22].

\subsection{POLE and POLD1 Mutations}

As POLE and POLD1 genes were missing from the TML assay, all samples were evaluated for mutations in these two genes using a custom next-generation sequencing panel. A germline Arg742Cys 
(exon 20; rs768004570) mutation was detected in the polymerase domain of POLE of patient 12 GL. This mutation was classified in the ClinVar database as of uncertain significance and annotated by PolyPhen software (genetics.bwh.harvard.edu/pph/) as probably damaging (Supplementary Materials Table S2).

\subsection{Microsatellite Instability}

Microsatellite instability (MSI) analysis was performed on all 16 samples comparing tumour and normal profiles of six polyA microsatellite markers. Two cases scored positive, 5 GL showed instability in 2/5 microsatellite markers (NR21, BAT26) and 12 GL in 4/5 microsatellites (NR21, NR24, BAT25, BAT26). These two cases had truncating or missense pathogenic mutations in $M S H 2$ and MSH6, respectively (Supplementary Materials Table S2). The remaining 14 cases had stable microsatellites including case $1 \mathrm{GL}$ with a MLH1 missense mutation reported as benign in the PolyPhen database (Supplementary Materials Table S2).

\subsection{Immunohistochemical Analysis of Mismatch Repair Proteins}

Three cases (1 GL, 5 GL, 12 GL) with mutations in MMR genes were immunostained for all four MMR proteins. Neoplastic cells of cases $5 \mathrm{GL}$ and $12 \mathrm{GL}$, which respectively harboured a double mutation in MSH2 (GLy164Glu and Val684Ter) and a mutation in MSH6 (Glu1322Ter), showed loss of the corresponding mutated protein (Figure 5) and positive staining for the other three proteins. Case 1 GL, harbouring the missense benign mutation in $M L H 1$, had positive immunostaining for all four MMR proteins.

\subsection{Ultra-Mutated IDH-wt GBMs Featured High Content of Giant Cells, MMR Protein Loss, and MSI}

Both ultra-mutated GBMs had at least 25\% of giant cells at histopathology (Figure 4) and TP53 mutations and pathogenic mutations in MMR genes (MSH2 and MSH6); case 12 GL also had POLE mutation. In addition, they had microsatellite instability as detected by MSI-PCR and MMR protein loss of expression at immunohistochemistry.

\subsection{Survival Analysis}

Overall survival ranged between 3 and 79 months (median: 19 months; mean 25 months); 8 (50\%) subjects died of the disease during follow-up.

At univariate analysis for tumour-specific survival, we tested: mutation in TP53; mutation in PTEN; amplification in EGFR; homozygous deletion in CDKN2A; presence of mutation in MMR genes MSH2 and MSH6; presence of TML over 9 mutations/Mb. The only significant genomic alteration associated with patients' survival was TML over 9 mutations/Mb $(p=0.04$; Figure 6$)$.

\subsection{Ultra-Mutated GBMs in the TCGA PanCancer Atlas}

Search for GBMs in the TGCA PanCancer Atlas through cBioPortal $[23,24]$ identified 266 cases with available information on exome mutations and patients age. Of these, 80 patients were $<55$ years (14 IDH-mut and 66 IDH-wt), and $186 \geq 55$ years (2 IDH-mut and $184 I D H$-wt). In these 266 GBMs, mutations ranged from 16 to 12, 189 per exome. Eight cases could be considered hyper-mutated and two ultra-mutated according to cut offs of $\geq 9$ muts/Mb for hypermutation and $>100$ muts/Mb for ultra-mutation, as defined by Campbell et al. [20]. In fact, the eight hypermutated cases had $\geq 275$ mutations per exome (range 275 to 1003) that correspond to $>9$ muts/Mb, assuming that an exome is $1 \%$ of the genome, that is $30 \times 10^{6} \mathrm{bp}$. The two ultra-mutated cases had 6910 and 12,189 mutations that correspond to $>200$ muts/Mb.

Of the eight hypermutated GBMs, four were from patients $<55$ years (4/80, 5\%) and included three $I D H$-wt- (377, 576, and 1003 mutations) and one IDH-mutated case (313 mutations), while four were $I D H$-wt from patients $\geq 55$ years $(275,284,343$, and 529 mutations). Among hyper-mutated GBMs, 
two cases had POLE mutations: an IDH-wt GBM from a 66 year old patient (TGCA-14-1795-01) which had 343 mutations and POLE Asp1123Asn mutation with unknown significance; a giant cell IDH-wt GBM from a 48 year old patient (case 19-1787-01) which had 576 mutations and POLE Ala399Val mutation reported of uncertain significance in the ClinVar database. None of the hypermutated cases had MMR mutations.

Only two cases were ultra-mutated and were IDH-wt from females aged 53 and 23 years, respectively: TCGA-19-5956 with 6910 mutations and TCGA-06-5416 with 12,189 mutations. Both cases harboured concomitant somatic mutations in POLE and MMR genes, namely, TCGA-19-5956 had two POLE (Arg1826Trp; Ala456Pro) and one MLH1 (Arg265Cys) missense mutations; TCGA-06-5416 had a POLE (Val411Leu), MSH2 (Lys871Asn), and MSH6 (Arg482Gln) missense mutations. Notably, case TCGA-19-5956 had been classified as giant cell GBM and case TCGA-06-5416 histologically showed multinucleated giant cells.

a

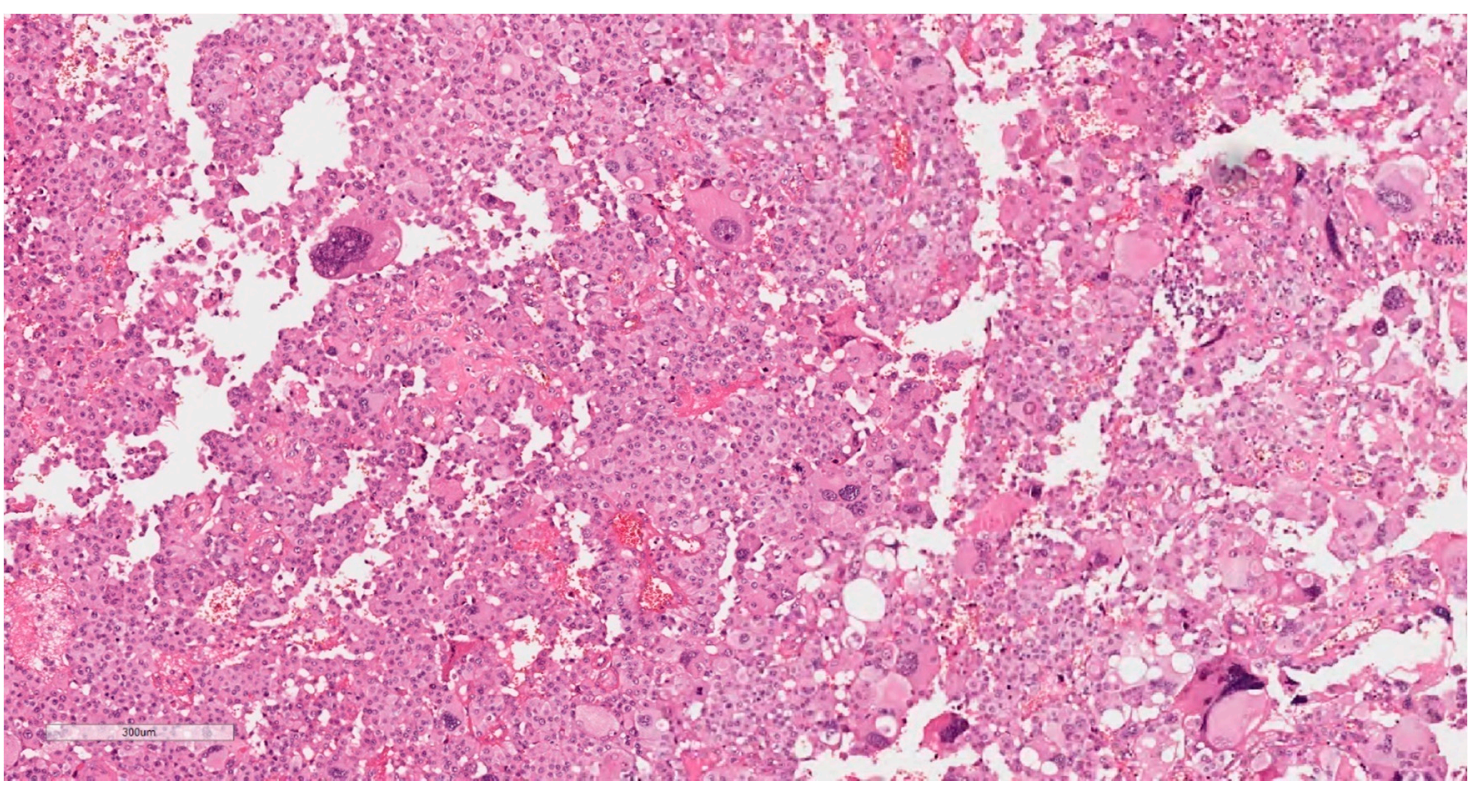

b

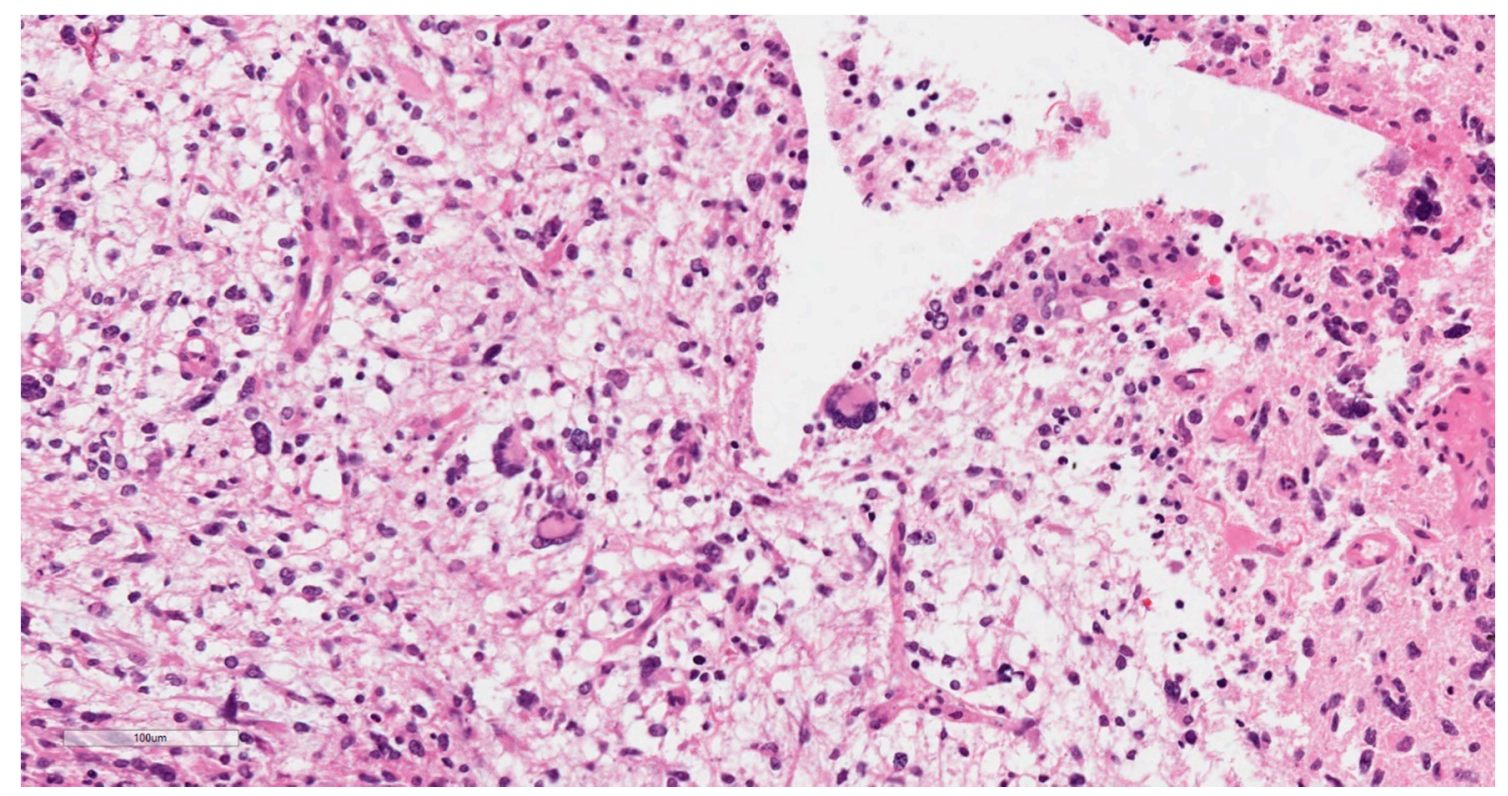

Figure 1. Histopathological aspects of ultra-mutated GBMs. Both cases 5 GL (a) and 12 GL (b) featured neoplastic giant cells bi- or multinucleated, which were uniformly dispersed throughout the tumour (a,b: original magnification, 200x). 


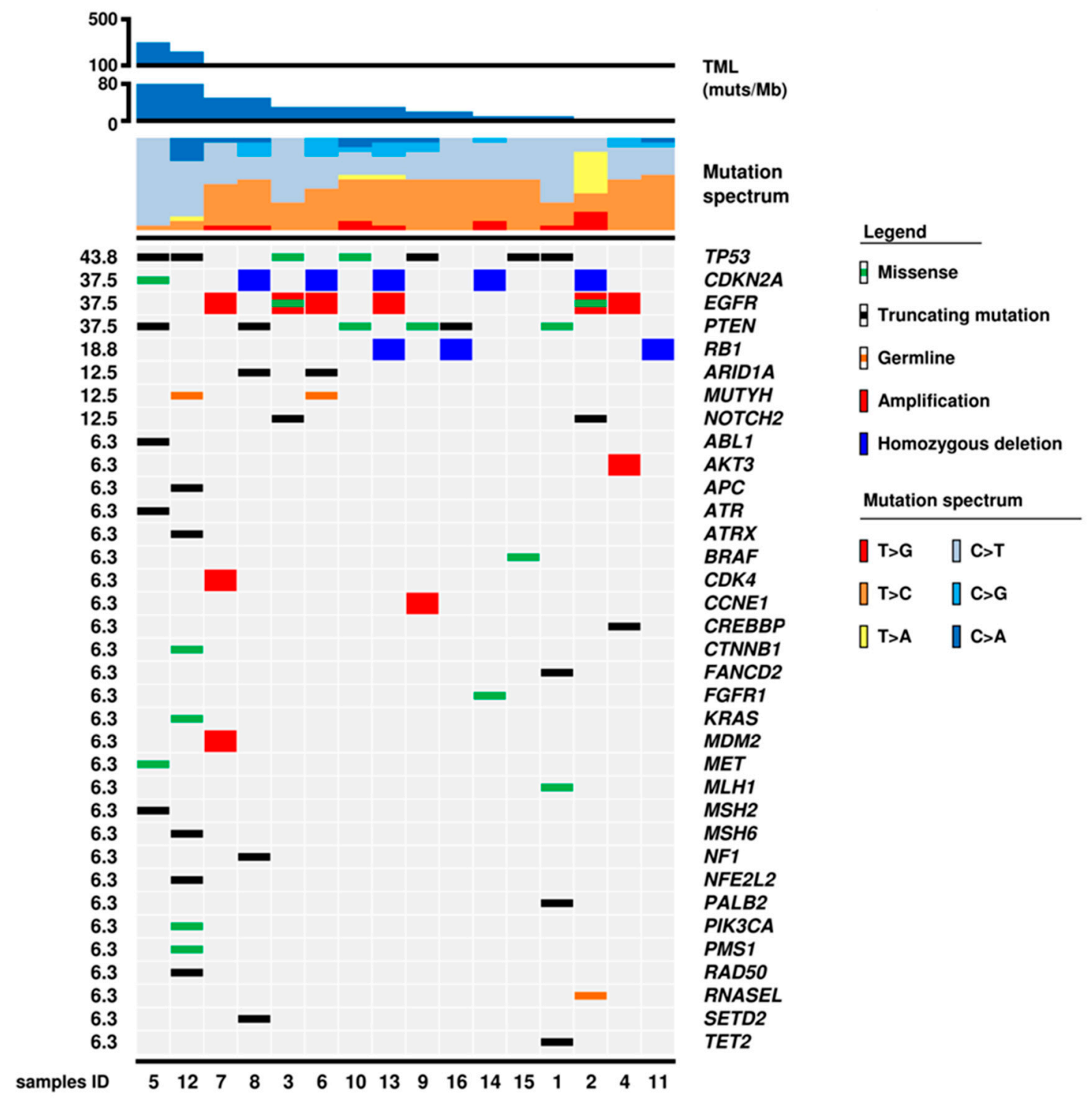

Figure 2. Genomic landscape of $16 \mathrm{IDH}$-wt glioblastomas. The matrix shows 36 genes that were altered at sequencing analysis; molecular alterations are annotated as illustrated in the panel below. Samples are ordered according to tumour mutational load (TML) from higher to lower values. The mutational spectrum is characterized by prevalent $\mathrm{T}>\mathrm{C}$ and $\mathrm{C}>\mathrm{T}$ transitions with low to absent contributions of $\mathrm{T}>\mathrm{A}, \mathrm{T}>\mathrm{G}, \mathrm{C}>\mathrm{G}$, and $\mathrm{C}>\mathrm{A}$ transversions. The proportion of $\mathrm{C}>\mathrm{T}$ over $\mathrm{T}>\mathrm{C}$ increased in parallel with the increase of TML. Case $12 \mathrm{GL}$ also showed a significant proportion of $\mathrm{C}>\mathrm{A}$ transversions. 


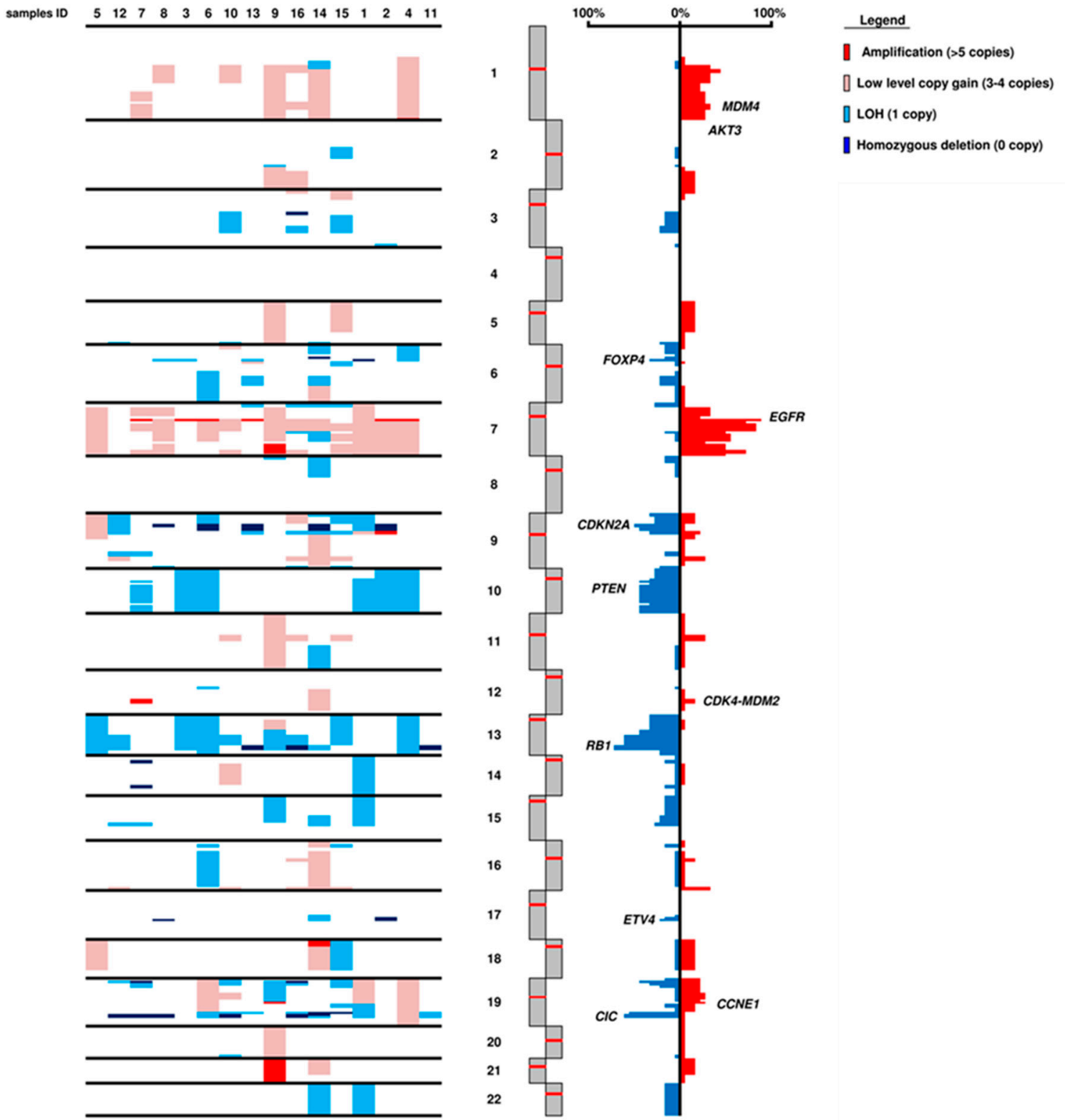

Figure 3. Chromosomal asset of $16 I D H$-wt glioblastomas. The panel summarizes copy number variation $(\mathrm{CNV})$ in whole chromosomes. Consensus of chromosome $\mathrm{CNV}$ is represented in red for copy gain events and in blue for loss events. 
TML: 168.02; MUTYH: Gly396Asp; MSH6: Glu1322Ter

$12 \mathrm{GL}$

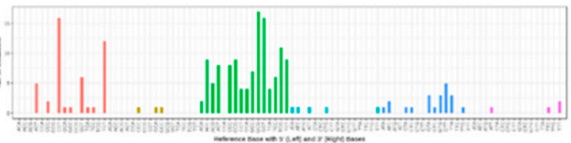

$1 G L$

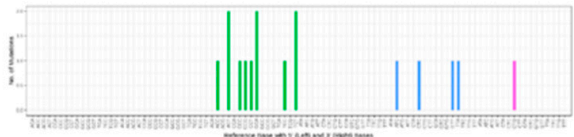

$3 G L$

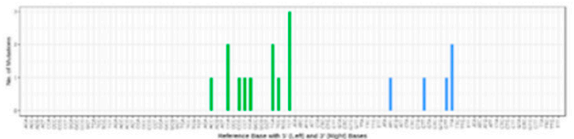

6GL

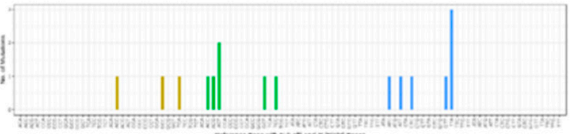

8GL

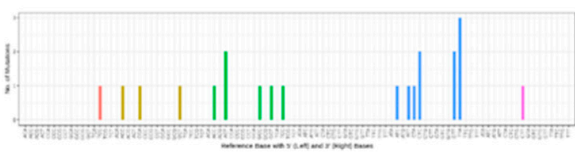

10GL

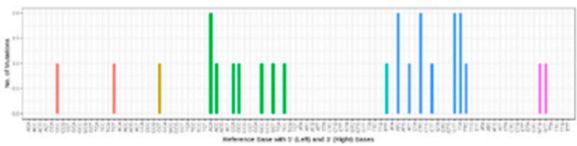

13GL

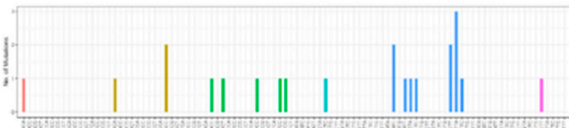

15GL

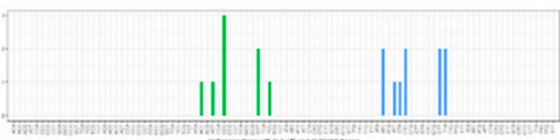

TML: 219.79; MSH2: Val684Ter

$5 \mathrm{GL}$

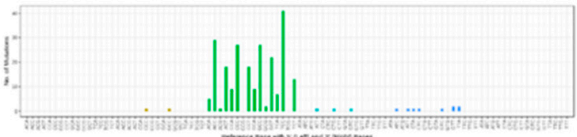

2GL

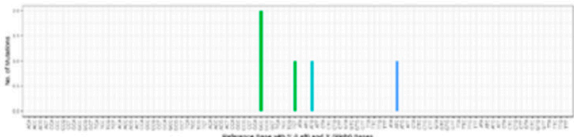

4GL

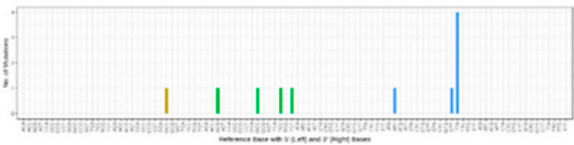

7GL

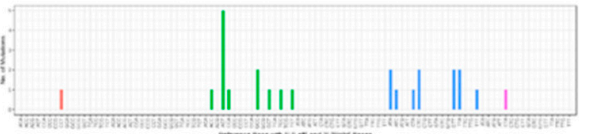

9GL

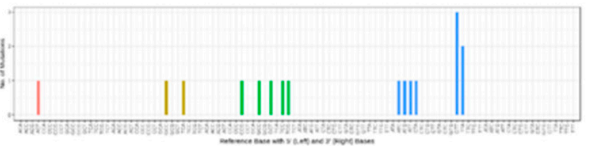

$11 \mathrm{GL}$

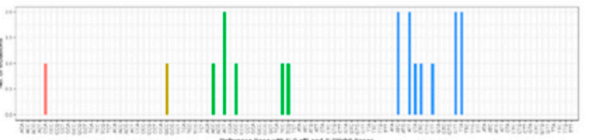

14GL

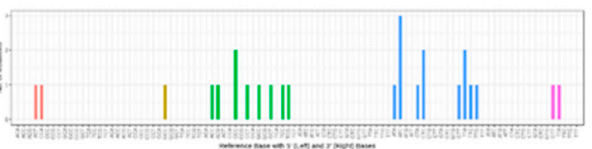

16GL

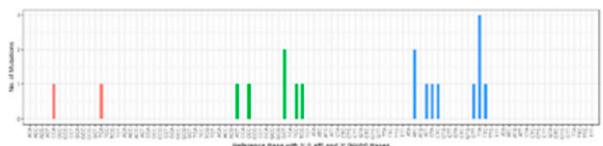

Substitution Type

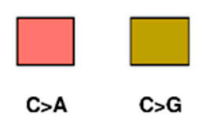

Figure 4. Mutational signatures of $16 \mathrm{IDH}$-wt glioblastomas. The signatures of somatic mutations (mutational spectrum) of individual tumours were obtained considering six major mutation classes: $\mathrm{C}$ $>\mathrm{T} ; \mathrm{C}>\mathrm{A} ; \mathrm{C}>\mathrm{G} ; \mathrm{T}>\mathrm{A} ; \mathrm{T}>\mathrm{C} ; \mathrm{T}>\mathrm{G}$. On top are the two ultra-mutated samples with indications of tumour mutational load (TML) and the peculiar molecular alterations. The mutational signature of case $12 \mathrm{GL}$ shows a predominance of $\mathrm{C}>\mathrm{T}$ over $\mathrm{T}>\mathrm{C}$ transitions, and four peaks of $\mathrm{C}>\mathrm{A}$ transversions in the trinucleotide contexts CCT, TCT, GCT, and ACT; this latter mutational pattern corresponds to signature SBS14 in the Catalogue Of Somatic Mutations In Cancer (COSMIC) version 3, and is associated to tumours with concurrent impairment of DNA mismatch repair and POLE proofreading functions (https://cancer.sanger.ac.uk/cosmic/signatures/SBS/) [25]. On the horizontal axis, the reference base with $5^{\prime}$ (left) and 3' (right) bases are shown. On the vertical axis, the number of mutations is shown. 


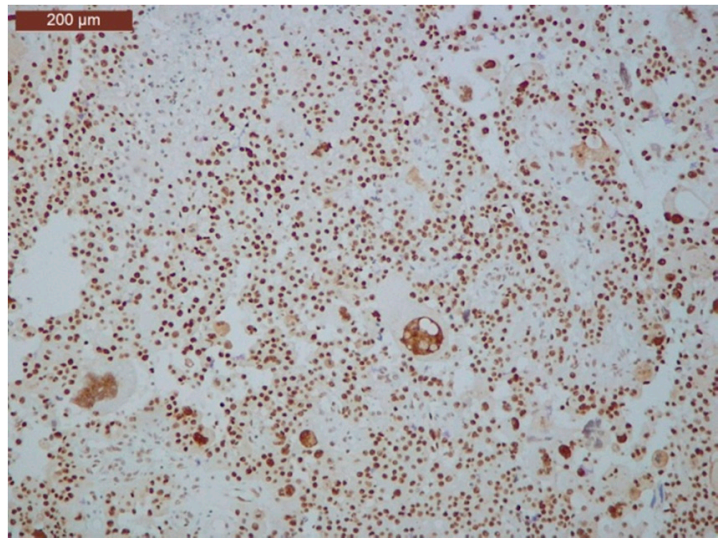

(a)

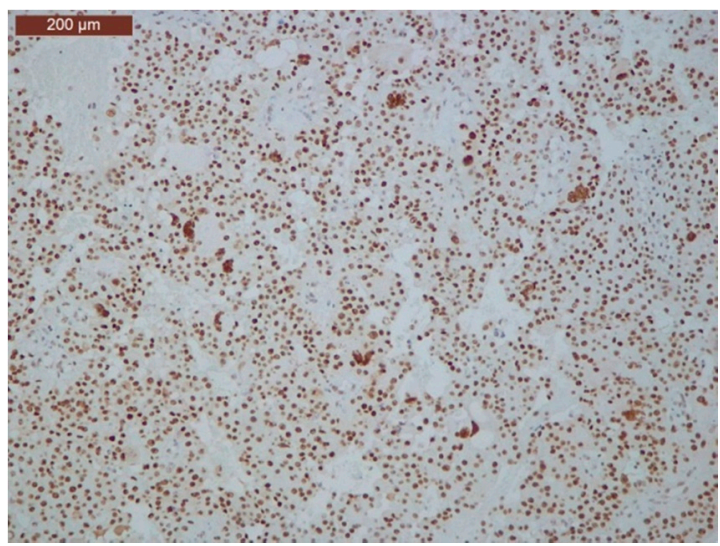

(c)

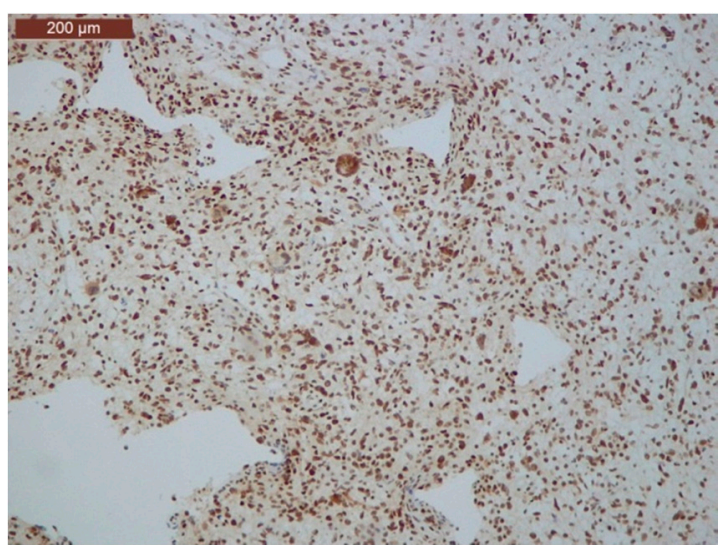

(e)

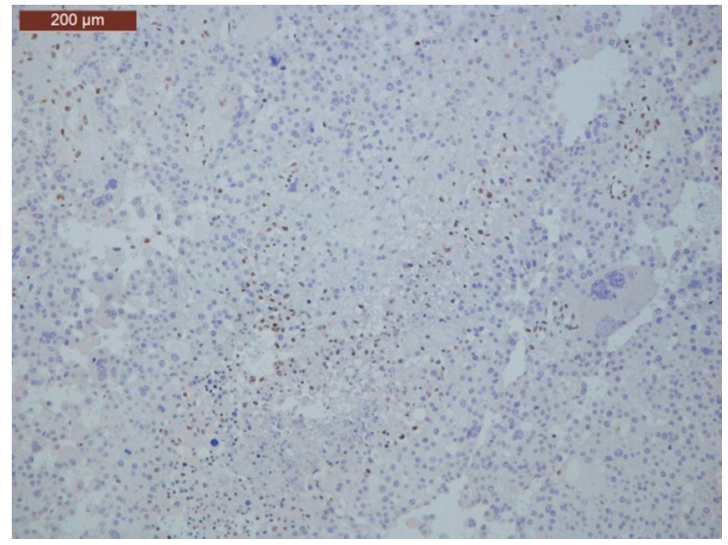

(b)

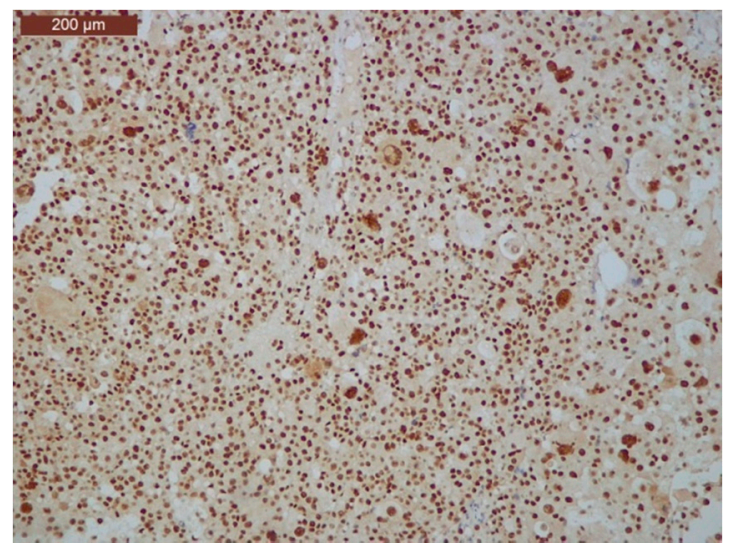

(d)

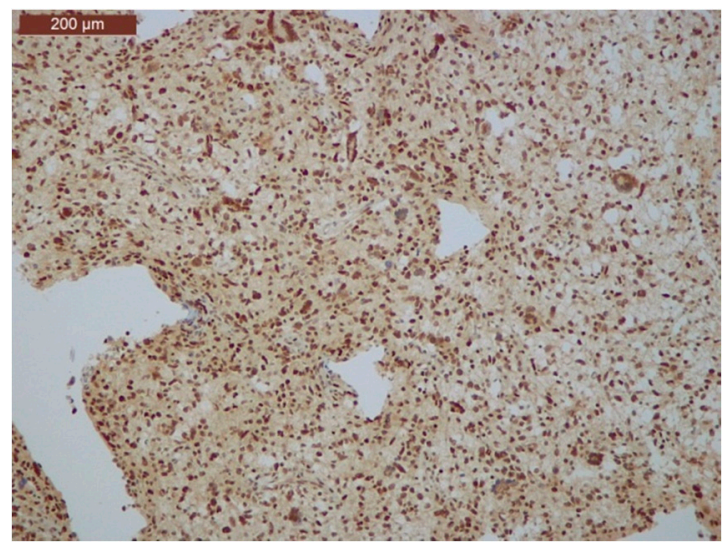

(f)

Figure 5. Cont. 


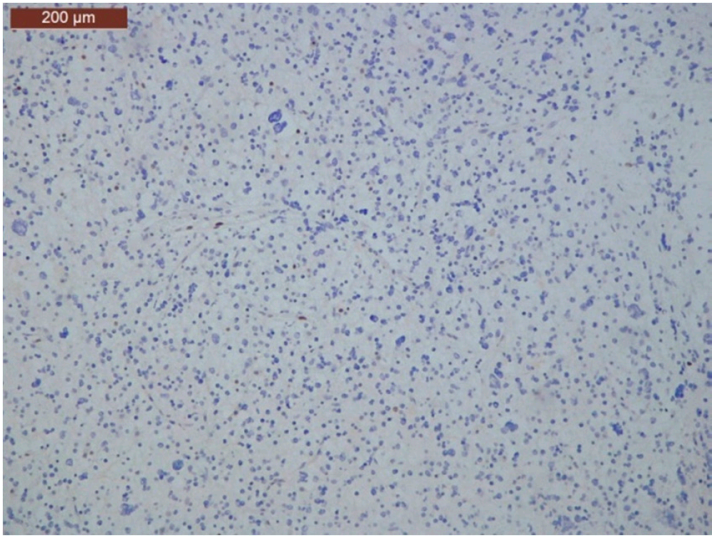

(g)

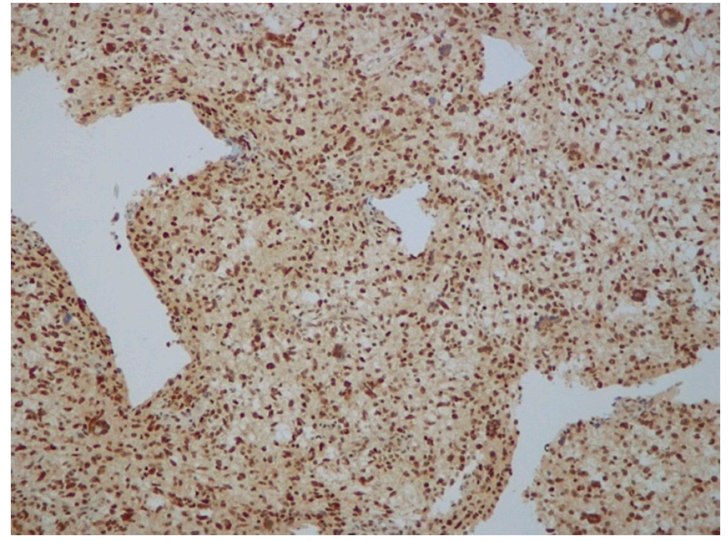

(h)

Figure 5. Immunohistochemical analysis of mutated mismatch repair genes in cases 5 GL (a-d) and 12 GL (e-h). Case 5 GL shows loss of MSH2 expression (b) and positive staining for MLH1, MSH6 and PMS2 (a,c,d) (original magnification, 200×). Case 12 GL shows loss of MSH6 expression (g) and positive staining for MLH1, MSH2, and PMS2 (original magnification, 200×) (e,f,h).

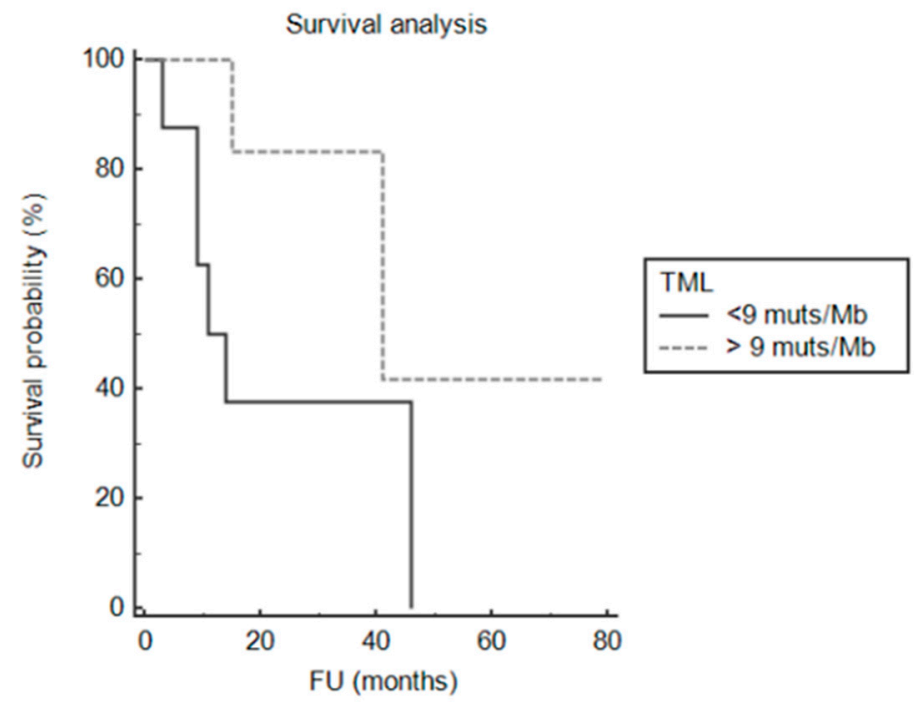

Figure 6. Impact of tumour mutational load on clinical outcome. Overall survival analysis showing that TML > 200 mutations/Mb was associated with better prognosis in IDH wild-type GBMs ( $p$-value $=0.036$ ).

\section{Discussion}

The results of our study on IDH-wt GBMs of adults < 55 years may be summarized as follows: (i) IDH-wt GBMs accounted for 14.8\% (16/108) of GBMs of this age group; (ii) TML in these 16 cases ranged between 5.66 and 219.79 mutations per megabase and, thus, 8 (50\%) GBMs were hypermutated ( $>9$ muts/MB) and two of those were ultra-mutated (> 100 muts/MB); (iii) 2 ultra-mutated GBMs had inactivating mutations in one MMR gene and MSI and they featured enrichment in giant cells; iv) one ultra-mutated GBM had also a germline POLE mutation previously undescribed as pathogenic; v) hyper-mutated GBMs had longer life expectancy.

The IDH-wt GBMs accounted for about $15 \%$ of GBMs $<55$ years in our hospital series and were characterized by frequent TP53 and PTEN mutation, EGFR gene amplification, CDKN2A homozygous deletion, gains of chromosome 7 and losses of chromosomes 10 and 13. All these figures are in line with those reported for IDH-wt GBMs [9-11,26-28].

As highlighted by Campbell et al., dissimilar thresholds and methodologies and the lack of an agreed definition of hypermutation are major issues in the study of TML [20]. To address these questions, 
they analysed TML in 78,452 adult and 2885 childhood cancers and established 9.9 and 9 muts/Mb as cut-offs for hypermutation in childhood and adult cancers, respectively, and 100 muts/Mb as the cut-off for ultra-mutation [20]. They also demonstrated excellent concordance among exome, genome, and two separate targeted panel sequencing consisting of 315 and 884 genes covering 1.1 and $3.25 \mathrm{Mb}$, respectively, for the assessment of hypermutation across different types of cancer [20]. By using those cut off values, 8 out 16 IDH-wt newly diagnosed GBMs in our cohort had $>9$ muts/Mb and should be considered hypermutated; two of those had $>100$ muts/Mb and should be classified as ultra-mutated. The frequency of hyper- and ultra-mutated tumours in our cohort ( $50 \%$ and $12.5 \%$, respectively) was higher than that reported in other cohorts of GBMs [16,17] and that found in GBMs within the TGCA PanCancer Atlas [20,21] However, it is in line with the high frequency of hypermutation in brain tumours found by Campbell et al. [20] among all hypermutated and ultra-mutated neoplasms. Difference with other studies may be due to the use of different methodologies in terms of analysed genes and type of mutations considered. In addition, we may hypothesize that the high frequency of ultra-mutated GBMs could depend on the selection criteria of our cohort (i.e., IDH-wt status and age below 55 years). Indeed, a similar frequency (11\%) of ultra-mutated cases was present among $I D H$-wt high-grade gliomas from patients $<55$ years old in the cohort of Erson-Omay et al. [29]. In addition, two ultra-mutated GBMs found in the Glioblastoma Multiforme TGCA PanCancer Atlas were IDH-wt and were from patients $<55$ years of age.

It is known that different mutagenic processes give rise to different combinations of mutation types, called signatures [30]. The mutational spectrum of our IDH-wt cohort was characterized by prevalent $\mathrm{T}>\mathrm{C}$ and $\mathrm{C}>\mathrm{T}$ transitions. Accordingly, two ultra-mutated GBMs had a signature characterized by a predominance of $C>T$ over $T>C$ transitions and low to absent contributions of $T>A, T>G$, and $\mathrm{C}>\mathrm{G}$ transversions signatures [30]. Interestingly, one of the cases also featured a high rate of $\mathrm{C}>\mathrm{A}$ transversions which presented four peaks in the trinucleotide contexts CCT, TCT, GCT, and ACT. This mutational signature overlaps COSMIC signature SBS14, which has been associated with tumours with concurrent impairments of POLE and MMR functions [21,22]. Fittingly, this GBM had MSI associated to a somatic MSH6 mutation and a germline mutation in the polymerase domain of POLE. This latter is the Arg742Cys substitution, which is reported as of uncertain significance in the ClinVar database. It involves the polymerase domain of POLE, and not the proofreading exonuclease one, different from POLE mutations which are commonly associated with hypermutation [19]. However, the association of Arg742Cys substitution with the COSMIC SBS14 mutational signature strongly suggests its pathogenicity [18]. Indeed, this signature is characteristic of tumours showing both MMR deficiency and POLE loss of function [18]. In addition, Campbell et al. [20] recently reported POLE driver mutations outside the exonuclease proofreading domain, suggesting that other domains might be responsible for proofreading. Interestingly, Erson-Omay et al. previously reported six ultra-mutated high-grade gliomas with a signature characterized by an increased proportion of $\mathrm{C}>\mathrm{A}$ transversions associated with somatic mutations in the POLE exonuclease domain and, in three cases, a germline MSH6 mutation [21].

Hypermutation in cancer is associated with defective MMR and/or POLE functions [16,20]. In particular, isolated MMR deficiency and microsatellite instability have been mostly restricted to hyper-mutated tumours (TML between 10 and 100 muts/Mb), while ultra-mutated tumours ( $>100$ muts/Mb) are microsatellite stable and POLE mutated. This study showed that hypermutation in GBM is not a unique feature of tumours with MMR genetic alterations. Indeed, fourteen hypermutated GBMs (six in our cohort and eight in the TGCA cohort) had no MMR mutations. In addition, it demonstrated that ultra-mutation can also be found in GBMs with MSI and isolated MMR deficiency. Indeed, one ultra-mutated GBM had $\mathrm{MSH} 2$ somatic mutation in the absence of POLE impairment. Accordingly, an ultra-mutated GBM with MSH6 somatic mutation, but not POLE mutation, was previously reported by Erson-Omay et al. [21]. In addition, MLH1 and MSH6 somatic mutations were recently found in one primary and four recurrent $I D H$-wt GBMs [11]. 
Erson-Omay et al. described a predominance of large and bizarre multinucleated giant cells in ultra-mutated GBMs with POLE mutations and they concluded that "somatic POLE altered ultra-mutated GBMs represent a subset of all giant cell GBM" [20]. In line with this observation, two ultra-mutated GBMs with POLE mutation in the TGCA PanCancer Atlas were histologically characterized by the presence of giant cells. However, both ultra-mutated GBMs in our cohort had giant cell enrichment and none had somatic POLE mutation, but rather germline POLE mutation or isolated MSH2 mutation. Besides, Vande Perre et al. recently reported POLE germline mutations in two patients with giant cell GBM [31]. Therefore, this study suggests that ultra-mutated GBMs might represent a subgroup of giant cell GBMs, which is not only characterized by POLE mutations. However, giant cell enrichment is not a unique feature of ultra-mutated GBMs and no ultra-mutated cases were found in a cohort of giant cell GBMs in a recent study [32].

Due to the small number of cases, we could not analyse whether there was a difference in survival length between hyper- and ultra-mutated GBMs. Although our findings should be interpreted with caution since multivariate survival analysis could not be performed due to the low number of patients, the hyper-mutated (including the ultra-mutated) GBMs in our cohort had significantly better prognosis than non-hypermutated tumours. Therefore, although IDH-wt GBMs are considered to have bad prognosis [4-6], our findings suggest that hyper-mutated GBMs may represent a less aggressive subgroup.

In addition to the prognostic significance, ultra-mutation in GBM might also have therapeutic implications. Indeed, a report suggests that ultra-mutated paediatric GBMs with germline MMR defects may have good response to immunotherapy [33]. Although limited data exist on the potential clinical benefit of immunotherapy in GBM, the evidence that $12.5 \%$ of $I D H$-wt GBM in adults $<55$ years are ultra-mutated may offer alternative therapeutic strategies in this age group.

\section{Materials and Methods}

\subsection{Cases}

A total of $16(14.8 \%) \mathrm{IDH}$-wt GBMs with available follow-up data were found among $108 \mathrm{GBMs}$ that had been surgically resected from patients between 18 and 54 years at the Unit of Neurosurgery of the Azienda Ospedaliera Universitaria "G. Martino" of Messina, Italy, between 2011 and 2018. In all cases, macroscopically complete surgical removal was achieved. None of the patients received preoperative therapy. All cases were formalin-fixed and paraffin-embedded (FFPE) for routine histological evaluation. Histological diagnosis was confirmed by independent revision of two pathologists before inclusion in the study. The IDH mutational status was firstly assessed by immunohistochemistry against IDH1 $\mathrm{R} 132 \mathrm{H}$, followed by IDH1/IDH2 sequencing in immuno-negative cases. The study was approved by the Ethics Committee of the Azienda Ospedaliera Universitaria (A.O.U) Policlinico Gaetano (G.) Martino (Messina, Italy) protocol number 47/19 of 2 May 2019. Informed consent was obtained from each patient.

\subsection{Mutational and Copy Number Variation Status of 409 Cancer Genes}

For each case, DNA was obtained from FFPE tumour and from matched non-neoplastic brain (surrounding the tumour that had been removed and showing no microscopic neoplastic infiltration) using 10 consecutive $4 \mu \mathrm{m}$ sections and the QIAamp DNA FFPE Tissue Kit (Qiagen) and qualified as reported elsewhere [34]. The Oncomine Tumour Mutational Load (TML) panel with next-generation sequencing assay (ThermoFisher) was used. The assay covers $1.65 \mathrm{Mb}$ of genomic space and includes all exons of 409 cancer-related genes.

Sequencing was performed on Ion Torrent platform using $20 \mathrm{ng}$ of DNA for each multiplex PCR amplification and subsequent library construction. The quality of the obtained libraries was evaluated by the Agilent 2100 Bioanalyzer on-chip electrophoresis (Agilent Technologies). Emulsion PCR to 
clonally amplify the libraries was performed with the Ion OneTouch ${ }^{\mathrm{TM}}$ OT2 System (ThermoFisher). Sequencing was run on the Ion Proton (ThermoFisher) loaded with Ion PI Chip v3.

Data analysis, including alignment to the hg19 human reference genome and variant calling, was done using Torrent Suite Software v.5.10 (TermoFisher). Filtered variants were annotated using a custom pipeline based on vcflib (https://github.com/ekg/vcflib), SnpSift [35], Variant Effect Predictor (VEP) [36], and NCBI RefSeq database. Additionally, alignments were visually verified with the Integrative Genomics Viewer (IGV) v. 2.3 [37] to further confirm the presence of identified mutations.

CNV was evaluated using OncoCNV v6.8 [38], comparing the BAM files obtained by sequencing of tumour samples with those obtained from blood samples. The software includes a multi-factor normalization and annotation technique enabling the detection of large copy number changes from amplicon sequencing data and permits to visualize the output per chromosome.

\subsection{Tumour Mutational Load and Mutational Signatures}

Tumour mutational load (TML) and mutational spectrum for each sample were evaluated using the Oncomine TML 5.10 plugin available on IonReporter software (ThermoFisher). Default modified parameters were used according to the manufacturer's protocol in order to exclude false positives due to the sequencing artefacts. In detail, a threshold of at least 20 reads and an allelic frequency of $10 \%$ of variant was used to perform mutation calling. In particular, TML was expressed as the number of mutations per megabase (muts/Mb), where mutations include non-synonymous missense and nonsense single nucleotide variants (SNVs), plus insertion and deletion variants (InDels) detected per megabase $(\mathrm{Mb})$ of exonic sequences.

The signatures of somatic mutations (mutational spectrum) of individual tumours were obtained considering six major mutation classes: $C>T(G: C>A: T) ; C>A(G: C>T: A) ; C>G(G: C>C: G)$; T > A (A:T > T:A); T > C (A:T > G:C); T > G (A:T > C:G) [27,28]. Mutational Signatures in Cancer (MuSiCa) software [39] was used to obtain specific signatures for each sample. The software used .vcf files to align the sequences to the hg19 human reference genome using targeted sequencing parameters. The different types of base-pair substitutions, comprising all non-synonymous missense and nonsense SNVs were normalized per $\mathrm{Mb}$ of exonic sequence. The percentage of each group in each sample was computed.

\subsection{Mutational Analysis of POLE and POLD1 Genes}

A next-generation DNA sequencing custom panel was designed to investigate the mutational status of POLE and POLD1 genes, which are not included in the TML panel. Sequencing was performed on Ion Torrent platform and data analysis was performed using the same pipeline used for TML panel.

\subsection{Microsatellite Instability Analysis}

MSI was tested by a fluorescent multiplex PCR exploiting the 5 mononucleotide microsatellites BAT25, BAT26, NR21, NR22, and NR24 [13]. The obtained amplicons were separated by capillary electrophoresis using the ABI Genetic Analyzer 3130XL platform (Applied Biosystems). Variations $\geq$ $3 \mathrm{bp}$ for BAT-25, NR21, and NR22 and $\geq 4 \mathrm{bp}$ for BAT-26 were considered as unstable.

\subsection{Immunohistochemistry of DNA Mismatch Repair Proteins}

Immunostaining was performed using the Bond Polymer Refine Detection kit (Leica Biosystems) in a BOND-MAX system (Leica Biosystems) on $4 \mu \mathrm{m}$ thick FFPE sections using the following primary antibodies purchased from DakoCytomation: mouse monoclonal clone ES05 against MLH1 at working dilution 1:30 and clone FE11 against MSH2 at working dilution 1:30; rabbit monoclonal clone EP49 against MSH6 at working dilution 1:100, and clone EP51 against PMS2 at working dilution 1:100. Normal cells within the samples were used as positive internal controls. 


\subsection{Statistical Analysis}

Overall survival was assessed by the Kaplan-Meier method, using the date of surgery as the entry data and length of survival until the patient's death as the endpoint. Patients who died of diseases independent from GBM were censored. The Mantel-Cox log-rank test was applied to assess the strength of association between disease-specific survival and molecular alterations as a single variable. A $p$-value $<0.05$ was considered as significant. All analyses were performed using MedCalc for Windows version 15.6 (MedCalc Software, Ostend, Belgium) and R v. 3.2.1

\section{Conclusions}

This study identified a molecular subgroup of ultra-mutated $I D H$-wt GBMs in adults $<55$ years, which was characterized histologically by the presence of at least $25 \%$ homogenously dispersed giant cells. Ultra-mutation and giant cells were not only associated with POLE mutations, but also with isolated defective MMR. Although this study has the limitation of a relatively low number of cases, it opens the perspective to potentially include these patients in immunotherapy clinical trials.

Supplementary Materials: The following are available online at http://www.mdpi.com/2072-6694/11/9/1279/s1, Table S1. Coverage detail of sequencing analysis performed. Table S2. List of somatic and germline mutations identified in 13 of the $16 \mathrm{IDH}$-wt glioblastomas, itemized by case and alphabetical order

Author Contributions: V.B. and M.S. contributed equally to this work. Conceptualization, V.B. and A.S.; Data curation, M.S., A.M., M.C., S.M.C., A.G., S.C. and C.G.; Formal analysis, M.S., A.M., M.L.P. and C.G.; Funding acquisition, A.S.; Investigation, M.L.P.; Methodology, A.M. and M.L.P.; Project administration, A.S.; Supervision, V.B. and A.S.; Writing—original draft, V.B. and M.S.; Writing—review and editing, V.B., M.C., S.M.C., A.G., S.C., C.G. and A.S.

Funding: The study was supported by Associazione Italiana per la Ricerca sul Cancro (AIRC $5 \times 1000$ n.12182 to AS).

Acknowledgments: We thank Alessandro Sorio and Claudia Parolini of Verona Hospital Trust for assistance in preparing materials.

Conflicts of Interest: The authors declare no conflict of interest.

\section{References}

1. Ostrom, Q.T.; Gittleman, H.; Truitt, G.; Boscia, A.; Kruchko, C.; Barnholtz-Sloan, J.S. CBTRUS Statistical Report: Primary Brain and Other Central Nervous System Tumours Diagnosed in the United States in 2011-2015. Neuro-Oncol. 2018, 20, iv1-iv86. [CrossRef] [PubMed]

2. Stupp, R.; Hegi, M.E.; Mason, W.P.; van den Bent, M.J.; Taphoorn, M.J.; Janzer, R.C.; Ludwin, S.K.; Allgeier, A.; Fisher, B.; Belanger, K.; et al. Effects of Radiotherapy with Concomitant and Adjuvant Temozolomide Versus Radiotherapy Alone on Survival in Glioblastoma in a Randomised Phase III Study: 5-year Analysis of the EORTC-NCIC Trial. Lancet Oncol. 2009, 10, 459-466. [CrossRef]

3. Stupp, R.; Mason, W.P.; van den Bent, M.J.; Weller, M.; Fisher, B.; Taphoorn, M.J.; Belanger, K.; Brandes, A.A.; Marosi, C.; Bogdahn, U.; et al. Radiotherapy Plus Concomitant and Adjuvant Temozolomide for Glioblastoma. N. Engl. J. Med. 2005, 352, 987-996. [CrossRef]

4. Hartmann, C.; Hentschel, B.; Wick, W.; Capper, D.; Felsberg, J.; Simon, M.; Westphal, M.; Schackert, G.; Meyermann, R.; Pietsch, T.; et al. Patients with IDH1 Wild Type Anaplastic Astrocytomas Exhibit Worse Prognosis than IDH1-mutated Glioblastomas, and IDH1 Mutation Status Accounts for the Unfavorable Prognostic Effect of Higher Age: Implications for Classification of Gliomas. Acta Neuropathol. 2010, 120, 707-718. [CrossRef] [PubMed]

5. Yan, H.; Parsons, D.W.; Jin, G.; McLendon, R.; Rasheed, B.A.; Yuan, W.; Kos, I.; Batinic-Haberle, I.; Jones, S.; Riggins, G.J.; et al. IDH1 and IDH2 Mutations in Gliomas. N. Engl. J. Med. 2009, 360, 765-773. [CrossRef]

6. Yao, Y.; Chan, A.K.; Qin, Z.Y.; Chen, L.C.; Zhang, X.; Pang, J.C.; Li, H.M.; Wang, Y.; Mao, Y.; Ng, H.K.; et al. Mutation Analysis of IDH1 in Paired Gliomas Revealed IDH1 Mutation was not Associated with Malignant Progression but Predicted Longer Survival. PLoS ONE 2013, 8, e67421. [CrossRef] 
7. Reuss, D.E.; Sahm, F.; Schrimpf, D.; Wiestler, B.; Capper, D.; Koelsche, C.; Schweizer, L.; Korshunov, A.; Jones, D.T.; Hovestadt, V.; et al. ATRX and IDH1-R132H Immunohistochemistry with Subsequent Copy Number Analysis and IDH Sequencing As a Basis for an "Integrated" Diagnostic Approach for Adult Astrocytoma, Oligodendroglioma and Glioblastoma. Acta Neuropathol. 2015, 129, 133-146. [CrossRef]

8. Stichel, D.; Ebrahimi, A.; Reuss, D.; Schrimpf, D.; Ono, T.; Shirahata, M.; Reifenberger, G.; Weller, M.; Hanggi, D.; Wick, W.; et al. Distribution of EGFR Amplification, Combined Chromosome 7 Gain and Chromosome 10 Loss, and TERT Promoter Mutation in Brain Tumours and Their Potential for the Reclassification of IDH-wt Astrocytoma to Glioblastoma. Acta Neuropathol. 2018, 136, 793-803. [CrossRef]

9. Aldape, K.; Zadeh, G.; Mansouri, S.; Reifenberger, G.; von Deimling, A. Glioblastoma: Pathology, Molecular Mechanisms and Markers. Acta Neuropathol 2015, 129, 829-848. [CrossRef]

10. Luois, D.N.; Ohgaki, H.; Wisteler, O.D.; Cavenee, W.K.; Ellison, D.W.; Figarella-Branger, D.; Perry, A.; Refeinberger, G.; von Deimling, A. WHO Classification of Tumours of the Central Nervous System; IARC: Lyon, France, 2016.

11. Korber, V.; Yang, J.; Barah, P.; Wu, Y.; Stichel, D.; Gu, Z.; Fletcher, M.N.C.; Jones, D.; Hentschel, B.; Lamszus, K.; et al. Evolutionary Trajectories of IDH(WT) Glioblastomas Reveal a Common Path of Early Tumourigenesis Instigated Years ahead of Initial Diagnosis. Cancer Cell 2019, 35, 692-704.e12. [CrossRef]

12. Ulgen, E.; Can, O.; Bilguvar, K.; Oktay, Y.; Akyerli, C.B.; Danyeli, A.E.; Yakicier, M.C.; Sezerman, O.U.; Pamir, M.N.; Ozduman, K. Whole Exome Sequencing-based Analysis to Identify DNA Damage Repair Deficiency as a Major Contributor to Gliomagenesis in Adult Diffuse Gliomas. J. Neurosurg. 2019, 1, 1-12. [CrossRef] [PubMed]

13. Luchini, C.; Bibeau, F.; Ligtenberg, M.J.L.; Singh, N.; Nottegar, A.; Bosse, T.; Miller, R.; Riaz, N.; Douillard, J.Y.; Andre, F.; et al. ESMO Recommendations on Microsatellite Instability Testing for Immunotherapy in Cancer, and Its Relationship with PD-1/PD-L1 Expression and Tumour Mutational Burden: A Systematic Review-based Approach. Ann. Oncol. 2019. [CrossRef] [PubMed]

14. Finocchiaro, G.; Langella, T.; Corbetta, C.; Pellegatta, S. Hypermutations in Gliomas: A Potential Immunotherapy Target. Discov. Med. 2017, 23, 113-120. [PubMed]

15. Schwartzentruber, J.; Korshunov, A.; Liu, X.Y.; Jones, D.T.; Pfaff, E.; Jacob, K.; Sturm, D.; Fontebasso, A.M.; Quang, D.A.; Tonjes, M.; et al. Driver Mutations in Histone H3.3 and Chromatin Remodelling Genes in Paediatric Glioblastoma. Nature 2012, 482, 226-231. [CrossRef] [PubMed]

16. Hodges, T.R.; Ott, M.; Xiu, J.; Gatalica, Z.; Swensen, J.; Zhou, S.; Huse, J.T.; de Groot, J.; Li, S.; Overwijk, W.W.; et al. Mutational Burden, Immune Checkpoint Expression, and Mismatch Repair in Glioma: Implications for Immune Checkpoint Immunotherapy. Neuro. Oncol. 2017, 19, 1047-1057. [CrossRef] [PubMed]

17. Johnson, A.; Severson, E.; Gay, L.; Vergilio, J.A.; Elvin, J.; Suh, J.; Daniel, S.; Covert, M.; Frampton, G.M.; Hsu, S.; et al. Comprehensive Genomic Profiling of 282 Pediatric Low- and High-Grade Gliomas Reveals Genomic Drivers, Tumour Mutational Burden, and Hypermutation Signatures. Oncologist 2017, 22, 1478-1490. [CrossRef] [PubMed]

18. Solomon, D.A.; Wood, M.D.; Tihan, T.; Bollen, A.W.; Gupta, N.; Phillips, J.J.; Perry, A. Diffuse Midline Gliomas with Histone H3-K27M Mutation: A Series of 47 Cases Assessing the Spectrum of Morphologic Variation and Associated Genetic Alterations. Brain Pathol. 2016, 26, 569-580. [CrossRef]

19. Riehmer, V.; Gietzelt, J.; Beyer, U.; Hentschel, B.; Westphal, M.; Schackert, G.; Sabel, M.C.; Radlwimmer, B.; Pietsch, T.; Reifenberger, G.; et al. Genomic Profiling Reveals Distinctive Molecular Relapse Patterns in IDH1/2 Wild-type Glioblastoma. Genes Chromosomes Cancer 2014, 53, 589-605. [CrossRef]

20. Campbell, B.B.; Light, N.; Fabrizio, D.; Zatzman, M.; Fuligni, F.; de Borja, R.; Davidson, S.; Edwards, M.; Elvin, J.A.; Hodel, K.P.; et al. Comprehensive Analysis of Hypermutation in Human Cancer. Cell 2017, 171, 1042-1056.e10. [CrossRef]

21. Catalogue of Somatic Mutations in Cancer (COSMIC). Version 3. Available online: https://cancer.sanger.ac. uk/cosmic/signatures/SBS/ (accessed on 20 August 2019).

22. Haradhvala, N.J.; Kim, J.; Maruvka, Y.E.; Polak, P.; Rosebrock, D.; Livitz, D.; Hess, J.M.; Leshchiner, I.; Kamburov, A.; Mouw, K.W.; et al. Distinct Mutational Signatures Characterize Concurrent Loss of Polymerase Proofreading and Mismatch Repair. Nat. Commun. 2018, 9, 1746. [CrossRef]

23. Hoadley, K.A.; Yau, C.; Hinoue, T.; Wolf, D.M.; Lazar, A.J.; Drill, E.; Shen, R.; Taylor, A.M.; Cherniack, A.D.; Thorsson, V.; et al. Cell-of-Origin Patterns Dominate the Molecular Classification of 10,000 Tumours from 33 Types of Cancer. Cell 2018, 173, 291-304.e6. [CrossRef] [PubMed] 
24. Liu, J.; Lichtenberg, T.; Hoadley, K.A.; Poisson, L.M.; Lazar, A.J.; Cherniack, A.D.; Kovatich, A.J.; Benz, C.C.; Levine, D.A.; Lee, A.V.; et al. An Integrated TCGA Pan-Cancer Clinical Data Resource to Drive High-Quality Survival Outcome Analytics. Cell 2018, 173, 400-416.e11. [CrossRef] [PubMed]

25. Mutational Signatures (V3-May 2019). Available online: https://cancer.sanger.ac.uk/cosmic/signatures/SBS (accessed on 20 August 2019).

26. Oh, J.E.; Ohta, T.; Nonoguchi, N.; Satomi, K.; Capper, D.; Pierscianek, D.; Sure, U.; Vital, A.; Paulus, W.; Mittelbronn, M.; et al. Genetic Alterations in Gliosarcoma and Giant Cell Glioblastoma. Brain Pathol. 2016, 26, 517-522. [CrossRef] [PubMed]

27. McNulty, S.N.; Cottrell, C.E.; Vigh-Conrad, K.A.; Carter, J.H.; Heusel, J.W.; Ansstas, G.; Dahiya, S. Beyond Sequence Variation: Assessment of Copy Number Variation in Adult Glioblastoma Through Targeted Tumour Somatic Profiling. Hum. Pathol. 2018. [CrossRef] [PubMed]

28. Brennan, C.W.; Verhaak, R.G.; McKenna, A.; Campos, B.; Noushmehr, H.; Salama, S.R.; Zheng, S.; Chakravarty, D.; Sanborn, J.Z.; Berman, S.H.; et al. The Somatic Genomic Landscape of Glioblastoma. Cell 2013, 155, 462-477. [CrossRef] [PubMed]

29. Erson-Omay, E.Z.; Caglayan, A.O.; Schultz, N.; Weinhold, N.; Omay, S.B.; Ozduman, K.; Koksal, Y.; Li, J.; Serin Harmanci, A.; Clark, V.; et al. Somatic POLE Mutations Cause an Ultramutated Giant Cell High-grade Glioma Subtype with Better Prognosis. Neuro. Oncol. 2015, 17, 1356-1364. [CrossRef] [PubMed]

30. Alexandrov, L.B.; Nik-Zainal, S.; Wedge, D.C.; Aparicio, S.A.; Behjati, S.; Biankin, A.V.; Bignell, G.R.; Bolli, N.; Borg, A.; Borresen-Dale, A.L.; et al. Signatures of Mutational Processes in Human Cancer. Nature 2013, 500, 415-421. [CrossRef] [PubMed]

31. Vande Perre, P.; Siegfried, A.; Corsini, C.; Bonnet, D.; Toulas, C.; Hamzaoui, N.; Selves, J.; Chipoulet, E.; Hoffmann, J.S.; Uro-Coste, E.; et al. Germline Mutation p.N363K in POLE is Associated with an Increased Risk of Colorectal Cancer and Giant Cell Glioblastoma. Fam. Cancer 2019, 18, 173-178. [CrossRef] [PubMed]

32. Shi, Z.F.; Li, K.K.; Kwan, J.S.H.; Yang, R.R.; Aibaidula, A.; Tang, Q.; Bao, Y.; Mao, Y.; Chen, H.; Ng, H.K. Whole-exome Sequencing Revealed Mutational Profiles of Giant Cell Glioblastomas. Brain Pathol. 2019. [CrossRef]

33. Bouffet, E.; Larouche, V.; Campbell, B.B.; Merico, D.; de Borja, R.; Aronson, M.; Durno, C.; Krueger, J.; Cabric, V.; Ramaswamy, V.; et al. ImmuneCheckpoint Inhibition for Hypermutant Glioblastoma Multiforme Resulting From Germline Biallelic Mismatch Repair Deficiency. J. Clin. Oncol. 2019, 34, 2206-2211. [CrossRef]

34. Simbolo, M.; Gottardi, M.; Corbo, V.; Fassan, M.; Mafficini, A.; Malpeli, G.; Lawlor, R.T.; Scarpa, A. DNA Qualification Workflow for Next Generation Sequencing of Histopathological Samples. PLoS ONE 2013, 8, e62692. [CrossRef] [PubMed]

35. Cingolani, P.; Patel, V.M.; Coon, M.; Nguyen, T.; Land, S.J.; Ruden, D.M.; Lu, X. Using Drosophila melanogaster as a Model for Genotoxic Chemical Mutational Studies with a New Program, SnpSift. Front. Genet. 2012, 3, 35. [CrossRef] [PubMed]

36. McLaren, W.; Pritchard, B.; Rios, D.; Chen, Y.; Flicek, P.; Cunningham, F. Deriving the Consequences of Genomic Variants with the Ensembl API and SNP Effect Predictor. Bioinformatics 2010, 26, 2069-2070. [CrossRef] [PubMed]

37. Robinson, J.T.; Thorvaldsdottir, H.; Winckler, W.; Guttman, M.; Lander, E.S.; Getz, G.; Mesirov, J.P. Integrative Genomics Viewer. Nat. Biotechnol. 2011, 29, 24-26. [CrossRef]

38. Boeva, V.; Popova, T.; Lienard, M.; Toffoli, S.; Kamal, M.; Le Tourneau, C.; Gentien, D.; Servant, N.; Gestraud, P.; Rio Frio, T.; et al. Multi-factor Data Normalization Enables the Detection of Copy Number Aberrations in Amplicon Sequencing Data. Bioinformatics 2014, 30, 3443-3450. [CrossRef] [PubMed]

39. Diaz-Gay, M.; Vila-Casadesus, M.; Franch-Exposito, S.; Hernandez-Illan, E.; Lozano, J.J.; Castellvi-Bel, S. Mutational Signatures in Cancer (MuSiCa): A Web Application to Implement Mutational Signatures Analysis In Cancer Samples. BMC Bioinformatics 2018, 19, 224. [CrossRef] [PubMed]

(C) 2019 by the authors. Licensee MDPI, Basel, Switzerland. This article is an open access article distributed under the terms and conditions of the Creative Commons Attribution (CC BY) license (http://creativecommons.org/licenses/by/4.0/). 